\title{
Efficient Edge Detection by Adapting Artificial Bee Colony Algorithm
}

\author{
By Ricardo Contreras Arriagada*, Ricardo Contreras Apablaza ${ }^{\dagger}$ \\ \& Maria Angélica Pinninghoff"
}

The problem of edge detection considers two stages: localisation and identification. Localisation is the search of pixels in an image; identification is the process of deciding whether a pixel belongs to an edge. The Canny edge detector technique is effective in identification; the process itself involves the analysis of every pixel that belongs to an image. Similarly, the artificial bee colony $(A B C)$ algorithm performs an efficient localisation of food sources by simulating the foraging behaviour of a honey bee swarm. In this proposal we integrate $A B C$ algorithm and Canny to create $A B C-E D$, an efficient edge detector algorithm that reduces the quantity of pixels to analyse, in order to detect its edges. We used the BSDS500 dataset for experimentation; our results show it is not necessary to analyse every pixel in the image to detect the same edges compared to Canny.

Keywords: $A B C-E D, A B C$ Algorithm, Imaging, Edge Detection, Canny Edge Detector

\section{Introduction}

In artificial vision and in image processing, edge detection deals with the localisation and identification of significant grey level variations in a digital image. Localisation relates to the search of points at a particular location in a grid of pixels. Identification relates to the process of deciding if a particular pixel belongs to an edge.

In image processing, an important number of edge detectors have been proposed exhibiting differences in terms of mathematic and algorithmic properties. In Ziou and Tabbone (1988) authors give an overview of research conducted in edge detection including properties of detectors, the methods for edge detection, the mutual influence between edge and detectors, and existing edge detectors and their implementation. One of the standard edge detection methods is proposed by Canny (1986), which offers an effective pixel identification by analysing every pixel in the image.

Artificial Bee Colony (ABC) algorithm, is a swarm intelligence algorithm that simulates the natural foraging behaviour of honey bees. Foraging behaviour of bees has a good balance between exploitation and exploration and uses communication mechanisms, such as the waggle dance, to explore and find new and better sources of food (Basturk and Karaboga 2006, Karaboga 2005). The term swarm is used to refer to any restrained collection of interacting individuals. A bee can discover different locations of sources of food. Different locations show different levels of nectar (better solutions). A bee chooses the location that holds the higher level of nectar. If a food source is exhausted during the foraging process, the exhausted source is

\footnotetext{
*Lecturer, University of Concepción, Chile.

'Research Fellow, University of Aberdeen, UK.

${ }^{+}$Lecturer, University of Concepción, Chile.
} 
abandoned, and bees explore new locations to find new sources of food (Cuevas et al. 2017).

$\mathrm{ABC}$ has been adapted for various problems in the area of image processing, in particular, in edge detection. A very interesting characteristic of $\mathrm{ABC}$ is that the probability of falling into local optimum is low, this is due to the combination of local and global search. In Benala et al. (2009), $\mathrm{ABC}$ was adapted for edge enhancement to improve visual perception of blurred images. Authors claim ABC algorithm is a powerful optimisation technique, they compare their results against results obtained using genetic algorithms techniques. In Parmaksizoglu and Alci (2011), the edge detection process is performed using cellular neural networks $(\mathrm{CNN})$. More specifically, the $\mathrm{ABC}$ algorithm was used to design the cloning template of a CNN. This work claims that the ABC- $\mathrm{CNN}$ approach gave better results, when compared to the classical edge detection techniques. In Yigitbasi and Baykan (2013), $\mathrm{ABC}$ was used to develop a method for edge detection without mask operator to compute the fitness of a pixel. Grey level values were used as the knowledge of nectar. In Deng and Duan (2014), a hybrid model of saliency-based visual attention and $\mathrm{ABC}$ algorithm was developed to narrow the searching region of an image. Authors developed an unmanned combat air vehicle (UCAV) able to recognise targets in complex noisy environments. A recent work (Ahmad et al. 2017) shows how bees' algorithm can be applied to health care problem. Biomedical signals are low amplitude, and low frequency signals affected by different noises. Noise can be triggered by different sources such as power line interference, or movements of recording electrodes. Authors claim the noise ratio can be reduced by designing the proper digital filters, using the $\mathrm{ABC}$ algorithm.

In this work we combine an effective identification mechanism (Canny), with an efficient search mechanism, (ABC algorithm). We develop an efficient edge detector algorithm for digital images based on Artificial Bee Colony and Edge Detector (ABC-ED).

This paper is structured as follows: the first section is the present introduction. The second section, Literature Review, introduces fundamental concepts related to the $\mathrm{ABC}$ algorithm and the edge detection problem. The third section, Methodology, describes the design of the ABC-ED algorithm. The fourth section shows the results obtained followed by section five, showing the conclusions.

\section{Literature Review}

Edge detection is a technique used in many (artificial) vision approaches and applications. Edges detection allows for a significant reduction in the amount of data to be processed. This is accomplished by filtering undesirable or non-significant data, while keeping the most important structural properties of an image, improving thus the overall performance.

Edge detection locates and identifies important changes in the intensities of neighbour pixels. These changes characterise the boundaries among objects 
or regions in an image. Of course, many problems arise during the edge detections process. Just to mention a few, problems may arise from the inclusion of false positives (false edge detection) and false negatives (nondetection of true edges). Similarly, high computational processing time and noise are also among additional issues to be considered within edge detection process.

Different methods have been proposed for edges detection. These methods can - most of time - be grouped into two main categories: based on first order derivative and based on second order derivative.

Edge detection methods based on first order derivative (gradient methods), are very sensitive to noise and produce thicker edges. Second order derivative methods (Laplacian based), are methods concerned with an automatised edge detection (still very noise-sensitive).

Classic methods based on first order derivative are (Prewitt 1970, Roberts 1965, Sobel and Feldman 1968). In these methods there is a convolution among the image and their corresponding masks or kernels, to generate a gradient image where edges are detected through the search of minimum intensity values in the neighborhoods of pixels. The decision to classify a pixel as an edge depends on the value of a threshold $\mu$. This is done for every pixel of the image.

The Laplacian is a 2-D isotropic measure of the second spatial derivative of an image. The Laplacian of an image highlights regions of rapid intensity change and is therefore often used for edge detection. The Laplacian is often applied to an image that has first been smoothed with something approximating a Gaussian smoothing filter in order to reduce its sensitivity to noise. The operator normally takes a single grey level image as input and produces another grey level image as output (Torre and Poggio 1986).

Canny is a very popular method that uses concept of both first and second order derivative. Canny edge detection is a multi-step algorithm that can detect edges along with noise reduction, because of the smoothing process of the image. Canny edge detector was developed in 1986 and is arguably the de facto standard edge detector. Canny satisfies the following properties: a) Low error rate: a good detection of existent edges only, b) good localisation: the difference between edge pixels detected and real edge pixels is minimum, and c) minimal response: only one detector response per edge.

In Canny, the first step is to smooth the image by using a Gaussian filter, then the gradient magnitude and direction are computed; the third step is for thinning edges through non-maximum suppression. Finally, a double thresholding process and edge tracking by hysteresis is applied.

In Thereshko (2000) the author modelled the food collection process of a honeybee colony as a collective action. The model considers three fundamental components: food source, employed bees and unemployed bees. It also restricts the collective behaviour to two different types: recruiting for a food source and the abandon of a depleted food source. In Karaboga (2005) the proposed ABC algorithm expands the model proposed in Thereshko (2000). More specifically, 
the model extends the categories to three groups: employed bees, onlooker bees, and scout bees - as it occurs nature.

In $\mathrm{ABC}$ algorithm, the food source represents a possible solution to an optimisation problem. The nectar of a food source corresponds to the quality (fitness) of a solution. The number of the employed bees or the onlooker bees is equal to the number of solutions in the population. At the first step, the $\mathrm{ABC}$ algorithm generates a randomly distributed initial population of $S N$ solutions (food source positions), where $S N$ denotes the size of employed bees and onlooker bees. After initialisation, the population of the positions (solutions) is subject to repeated cycles, $C=\{1,2, \ldots$, Maximum Number of Cycles $(M C N)\}$, of the search processes of the employed bees, the onlooker bees and the scout bees. An employed bee produces a modification on the position (solution) in its memory depending on the local information (visual information) and tests the amount of nectar (fitness value) of the new source (new solution). If the nectar amount of the new one is higher than that of the previous one, the bee memorises the new position and forgets the old one. Otherwise she keeps the position of the previous one in her memory. After all employed bees complete the search process, they share the nectar information of the food sources and their position information with the onlooker bees. An onlooker bee evaluates the nectar information taken from all employed bees and chooses a food source with a probability related to its nectar amount. As in the case of the employed bee, she produces a modification on the position in her memory and checks the nectar amount of the candidate source. If the nectar is higher than that of the previous one, the bee memorises the new position and forgets the old one Karaboga and Akay (2009).

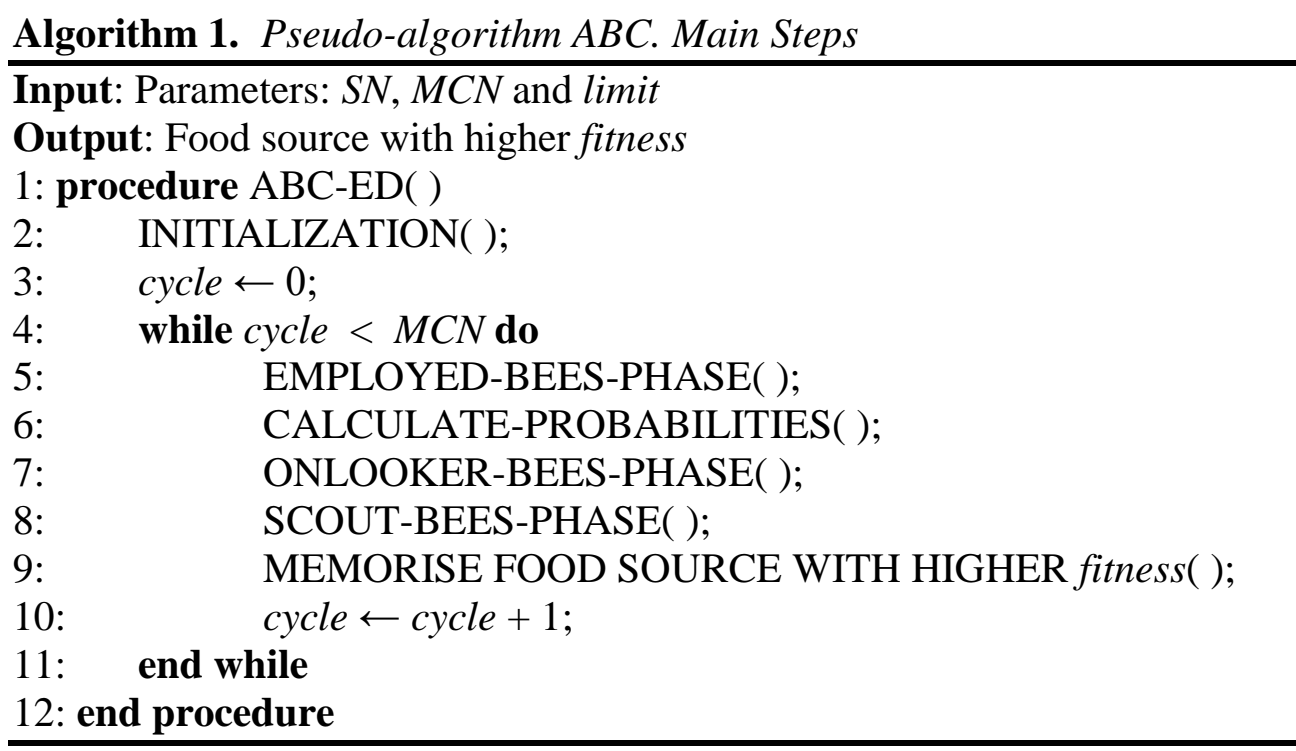

The general structure of the algorithm is shown in Algorithm 1. SN is the number of food sources, $M C N$ is the maximum number of cycles and limit is the number of tests to abandon a food source which cannot be improved. In every cycle of the algorithm $S N$ food sources are considered. 


\section{Methodology}

The input image $I$ is represented by a matrix of $r$ rows and $c$ columns. Every element in the matrix corresponds to a pixel in $I$. The value of each element indicates the intensity of the pixel in a grey scale. More specifically, a value of 0 corresponds to a black pixel, a value of 255 corresponds to a white pixel, and the values within this range (i.e. 0 - 255) correspond to the black - white chromatic colour of the pixel.

In addition, a pixel also represents a possible source of food for bees. The fitness of a pixel $\nabla(\mathrm{x}, \mathrm{y})$, is computed considering:

The intensity value of a pixel $I(x, y)$, in location $(x, y)$. The gradient magnitude $G_{x}(x, y)$ in horizontal direction of $I(x, y)$. And the gradient magnitude $G_{y}(x, y)$ in vertical direction of $I(x, y)$ as described in (Canny 1986).

The components $G_{x}(x, y)$ and $G_{y}(x, y)$ are combined to obtain the fitness value of pixel $(x, y)$, see Equation 3.1, fitness value of a pixel.

$$
\nabla(x, y)=G(x, y)=\sqrt{G x(x, y)^{2}+G y(x, y)^{2}}
$$

The quality of a food source is given by the difference of brightness intensities among a particular pixel and their neighbours. The greater the difference the higher the fitness (profitability of a food source).

The algorithm takes into account food sources with a fitness greater or equal than $\mu_{\text {min }}$. In other words, a pixel is classified as a food source (edge) if its fitness is greater or equal to the threshold $\mu_{m i n}$. This condition reduces considerably the number of pixels to be computed and therefore the overall execution time. Fitness computation of a pixel is performed once, at the moment of the food source creation.

The main steps of the ABC-ED are shown in Algorithm 2. The algorithm is a modified version of the original Canny procedure. More specifically, the proposed algorithm introduces a pixel selection mechanism based on bee colony. The localisation mechanism reduces the number of pixels to be processed in the original Canny procedure. Thus, reducing the total amount of edge thinning, double thresholding, and hysteresis performed.

The parameters of the algorithm are as follows. Input image matrix IM. Maximum $\mu_{\max }$ and minimum $\mu_{\min }$ thresholds parameters used by Canny. The initial population of food sources $S N$ computed as $S N=\sqrt{r * c}$ [22]. An

arbitrary number $M C N$ representing the number of cycles associated to the search of food sources.

In addition, limit $\in[0,8]$ represents the maximum number of trials to explore the neighbourhood of a pixel. A value of eight indicates all neighbours have been analysed, see Moore (1969).

Finally, $\varepsilon$ is a parameter $\varepsilon \in[0,100]$ used during the scout bees' phase to guide the search process of new food sources. 


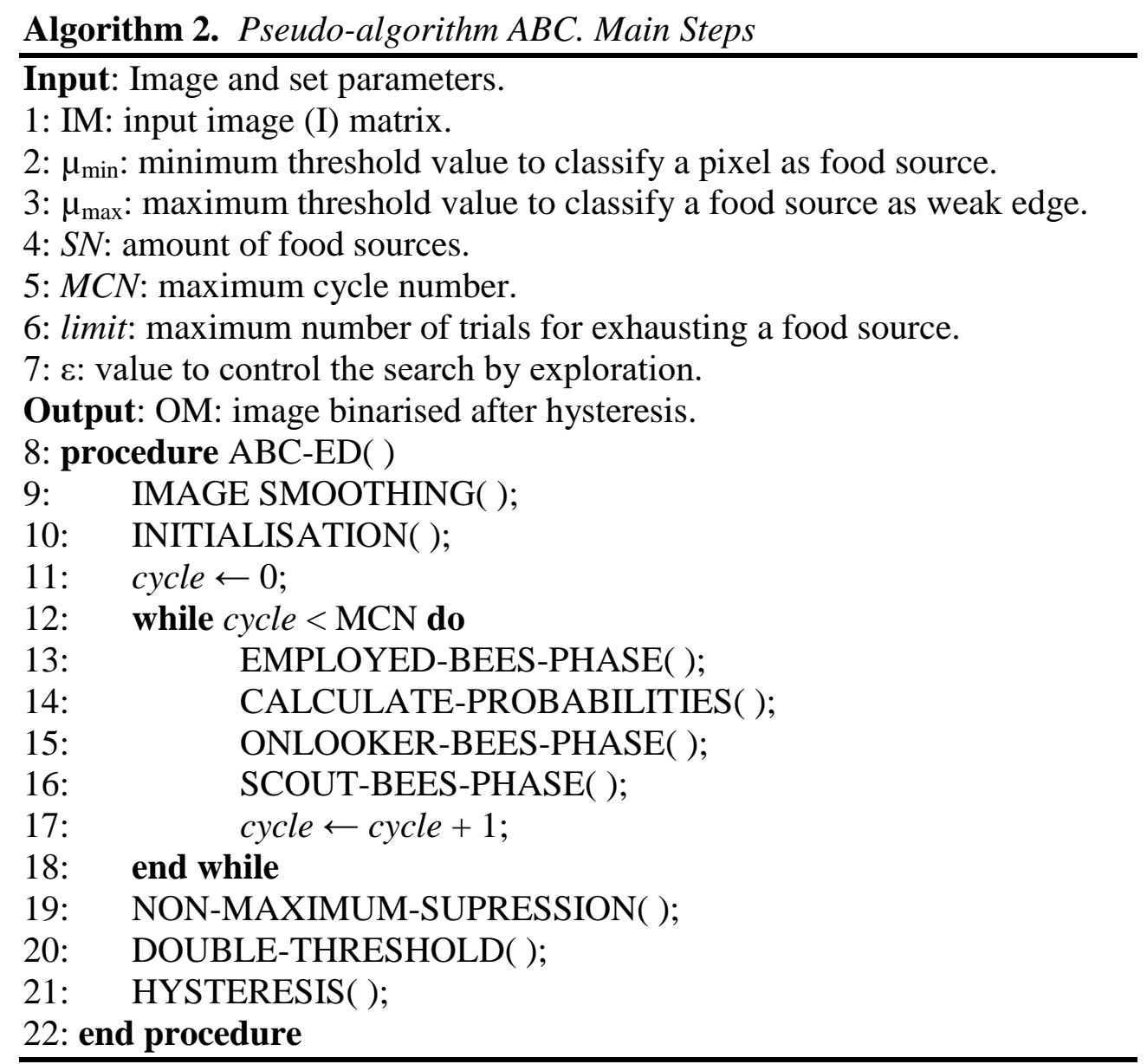

Four disjoint sets are defined to represent the possible states of a food source (i.e. a pixel). AFS represents the active food sources; every active food source is associated with one bee. IFS represents the inactive food sources; the sources in this category were replaced by a neighbour in the employed bees phase or in the onlooker bees phase. EFS represents the exhausted food sources for which all their neighbours have been examined. Finally, $R P$ represents all the non-food sources. More specifically, food sources in this category have a gradient magnitude lower than $\mu_{\min }$ (they will not be considered in future computations).

The output image is a binarised one. The image depicts the edges of the image in white, non-edge pixels are left in black.

In what follows, we provide a description for the different components of the Algorithm 2.

Edge detectors are affected by noise in the image. The noise must be filtered to prevent false edge detection. We apply a Gaussian filter to reduce the effect of noise on the edge detectors (Canny 1986). This process is known as smoothing.

In Initialisation, a population of $S N$ food sources is created by random pixel selection. This process is accomplished by randomly selecting $S N$ 
different $(x, y)$ pairs which represent different locations in matrix IM. For every selected pixel, the corresponding fitness is computed. If the fitness value is equal or greater than $\mu_{\min }$, the pixel is a candidate to be a source food (i.e. an edge) and added to $A F S$. If the fitness value is less than $\mu_{\min }$, the pixel is added to $R P$.

In Employed Bees Phase, every employee bee $k, k \in\{1,2, \ldots, S N\}$ is assigned to a food source $f_{k} \in A F S$. Only one employee bee is associated to a particular $f_{k}$. For each food source $f_{k}$ in $A F S$, a candidate neighbour $n f_{k}$ is randomly chosen. This random selection considers only those neighbours that remain unvisited. Once they are visited their fitness is computed.

If the computed fitness is less than $\mu_{m i n}$, the pixel is added to $R P$ and the value of limit $_{k}$ is increased in one unit. Otherwise, the computed fitness is assigned to $n f_{k} ; n f_{k}$ replaces the previous $f_{k}$ food source, and the value of limit $_{k}$ is set to zero.

The selection of neighbours in the ABC-ED algorithm differs from the selection of neighbours in $\mathrm{ABC}$ algorithm. In $\mathrm{ABC}$, the condition to replace the current food source, $\left(f_{k}\right)$ is that the fitness value of $n f_{k}$ is greater than the fitness value of $f_{k}$. In ABC-ED the condition to replace the current food source is that its neighbour $n f_{k}$ has a fitness value greater or equal to $\mu_{\min }$. If the replacement occurs, $f_{k}$ becomes inactive and added to IFS. If $f_{k}$ does not have neighbours to visit, it is said to be exhausted and it is added to EFS.

Employed bees share their food source information with onlooker bees waiting in the hive. Onlooker bees choose their food sources depending on the acquired information. More specifically, onlooker bees choose a food source depending on the probability values calculated from the fitness values (provided by employed bees).

In Calculate Probabilities, for each food source $f_{k}$ in $A F S$ the algorithm proceeds as follows (according to the information supplied by the employed bees). The probability $p\left(f_{k}\right)$ of selecting a food source $f_{k}$ is computed using expression shown in Equation 3.2, where $f i t\left(f_{k}\right)$ represents the value for the fitness of food source $f_{k}$. This creates an $A V L$ Roulette wheel selection (Baeck et al. 2000). Search of a food source is based on probability. A selfbalancing binary search tree $A V L$ (Adelson-Velskii and Landis 1962) represents the waggle dance of the employed bees. Equation 3.2 shows the probability of selecting a food source.

$P(f k)=f i t(f k) / \sum_{k=1}^{S N} f i t(f k)$

(Equation 3.2)

In onlooker Bees Phase, SN food sources are chosen stochastically using the $A V L$ Roulette. A food source can be chosen more than once in the same phase of the onlooker phase cycle. The greater the value of $p\left(f_{k}\right)$ and the greater the number of food source neighbours; the higher the probability an onlooker bee chooses $f_{k}$ as a food source. Once the food source is chosen, the onlooker bees proceed like the employed bees' phase already described. 
For each food source, it must be checked whether is exhausted. If the food source is exhausted, it is removed from $A F S$ and then added to $E F S$, and the onlooker bee becomes scout bee. This new scout bee starts the search for new food sources.

In scout Bees Phase, the replacement is chosen between New Random Exploration and Inactive Food Source mechanisms. The New Random Exploration mechanism randomly creates a new food source $\left(f_{\text {new }}\right)$ that is added to $A F S$ and replaces the former $f_{k}$ food source. The Inactive Food Source mechanism creates a new food source by removing the former source from the $I F S$ set, replacing the former $f_{k}$ food source, and adding $f_{\text {new }}$ to $A F S$. A FIFO strategy is used to remove a food source from IFS. The reason behind this strategy is that early added sources possess higher probabilities of having neighbours not yet examined.

The parameter $\varepsilon$ is used to guide the replacement mechanism. An $\varepsilon=$ 100 indicates the replacement mechanism is New Random Exploration. An $\varepsilon$ $=0$ indicates the replacement mechanism is Inactive Food Source. Similarly values within $\varepsilon \in$ ]0,100[ indicate the tendency of selecting New Random Exploration or Inactive Food Source. A value close to 0 is associated with a higher probability that the replacement mechanism is Inactive Food Source. A value close to 100 is associated with a higher probability that the replacement mechanism is New Random Exploration.

Once the bees' cycle is finished, a new FSP is created. FSP is the union of all the sets that contain a pixel (i.e. a candidate to be an edge) $F S P=A F S$ $\cup I F S \cup E F S$.

The expected output is a binarised image obtained by applying non maximum suppression, double thresholding, and hysteresis to every pixel in FSP. Non maximum suppression is a technique used to thin an edge. After gradient calculation, an extracted edge can still be quite blurred. Non maximum suppression avoids the blurring by setting all the gradient values to 0 with the exception of the local maximal. Thus, a location with the sharpest change of intensity value is easily identifiable.

Double-thresholding is a process that determines how strong an edge is. The process considers two threshold values: $\mu_{\max }$ and $\mu_{\min }$. These values are empirically determined and depend on the characteristics of the input image. A pixel with a fitness equal or greater than $\mu_{\max }$ is marked as a strong edge pixel. A pixel with a fitness equal or greater than $\mu_{\min }$ but lower than $\mu_{\max }$ is marked as a weak edge pixel. The process of hysteresis analyses the impact of a strong pixel. More specifically, it analyses the neighbours of a strong pixel and it changes their states if they are weak pixels. This process is repeated for each new strong marked pixel. Only strong pixels are considered edge pixels [6].

For integrating the $A B C$ algorithm with the Canny algorithm involved the following changes in $A B C$.

- A pixel is replaced when the fitness of the neighbour is higher than umin. This is possible because the proposed algorithm is not searching 
for an optimum value, but for a set of solutions satisfying a given constraint.

- Four set were considered to represent particular states associated to a food source. a) AFS is associated to active sources (recruitment), b) IFS and $E F S$ are associated to inactive and exhausted sources respectively, and c) $R P$ associated to non-food source. These sets are used to avoid repeating computation of the fitness. These sets also help in the intensification of the search of edge pixels.

- In the stage of scout bees, the process of seeking for a new source introduces a parameter $\varepsilon$. This parameter is used to choose between two alternative searching processes: an exploratory search and an exploiting search. The exploratory search realises a random search on the solutions space.

- The exploiting search, re-visit pixels that have still non-explored neighbours. The result is an extensive and intensive search guided by the value of $\varepsilon$ and the random value which, compared with $\varepsilon$ allows to decide between intensive and extensive search.

\section{Results}

For testing, we used The Berkeley Segmentation Dataset and Benchmark (BSDS500) (Martin et al. 2001). The dataset contains 500 images. For each image, there are about five segmented images in grey scale. In addition, for every segmented image there is a ground truth image. The ground truth image contains the edges that correspond to an image. The dataset contains a total of 2696 segmented images. For every segmented image, threshold values $\mu_{\min }$ and $\mu_{\max }$ are computed in four different ways: Mean (Glasbey 1993), Median, MATLAB (given by the function edge using Canny on MATLAB), and Otsu (1979). In average, the computed threshold values are similar, except for MATLAB which produces significant lower values.

Computing the correct threshold values is critical. If the minimum threshold is too small, several pixels could be considered potential food sources, leading to an increment in the processing time. On the other hand, if the maximum threshold is too high, pixels that correspond to edges, may not be considered as such. In average, best results are obtained using Otsu.

Performance is measured as the percentage of pixels necessary to analyse, with respect to the total pixels of the image, to achieve the same results obtained by Canny. We use Hamming Distance (HD) (Hamming 1950), to get the difference between two binarised matrices. More specifically, we use $H D$ to analyse the difference be- tween the image obtained from the ABC-ED algorithm, and the corresponding ground truth image.

Table 1 shows the results for different groups of images. The first three rows in the table consider an analysis of up to $25 \%$ of the total of pixels in the image. The fourth row considers an analysis that covers from $96 \%$ to $100 \%$ of the total amount of pixels in the image. A typical image has a size of $369 \times 432$ pixels. 
Table 1 shows the output for the images. For simplicity images are grouped into first group (the first three rows in Table 1) and second group (the fourth row in Table 1). The first group contains $44.7 \%$ of the images in the database, while the second group contains $49.26 \%$ of the images in the database. The remaining $6 \%$ correspond to images related to analysis ranging from $26 \%$ to $95 \%$. This correspond to less than 650 images and are not included in the overall analysis.

Table 1. Summary of Output Results

\begin{tabular}{|c|c|c|c|}
\hline Quantity & PA & MCN & $\Delta$ GT \\
\hline 3,224 & 15 & 82 & 1.03 \\
\hline 1,079 & 20 & 122 & 1.54 \\
\hline 518 & 25 & 131 & 1.79 \\
\hline 5,312 & 100 & 151 & 2.01 \\
\hline
\end{tabular}

In Table 1, Quantity refers to the number of output images. $P A$ denotes the maximum percentage of pixels analysed. $M C N$ represents the average number of cycles needed to detect the edges Canny detects. $\triangle \mathrm{GT}$ corresponds to the average difference between the output image and its ground truth image.

The first row considers 3,224 output images (Quantity). For each image up to $15 \%$ of pixels are analysed $(P A)$. In average, the algorithm executes 82 cycles for obtaining the output image $(M C N)$. The average difference between the output image and the ground truth image is about $1 \%(\Delta \mathrm{GT})$. The same applies to rows 2, 3 and 4 . It can be seen that the algorithm requires few pixels to analyse and cycles to compute to obtain an image that is no significantly different from the ground truth image.

Table 2. The Best Results - Ground Truth Differences

\begin{tabular}{|l|c|c|c|}
\hline Image & Thresholding & PA (\%) & $\Delta$ GT (\%) \\
\hline 1 & Median & 40.28 & 0.09 \\
\hline 2 & MATLAB & 40.35 & 0.09 \\
\hline 3 & Mean & 41.29 & 0.09 \\
\hline 4 & Otsu & 47.77 & 0.09 \\
\hline 5 & MATLAB & 40.02 & 0.1 \\
\hline 6 & Median & 40.58 & 0.1 \\
\hline 7 & Mean & 40.82 & 0.1 \\
\hline 8 & Otsu & 50.27 & 0.1 \\
\hline 9 & Median & 39.22 & 0.13 \\
\hline 10 & Mean & 40.65 & 0.13 \\
\hline
\end{tabular}

Table 2 shows our results. The results are ordered according to the average difference between the output image and the ground truth image $(\Delta \mathrm{GT})$. The first row (lowest difference) shows that the output image presents a difference of $0.09 \%$ with respect to the ground truth image. The quantity of pixels analysed corresponds to $40.28 \%$ of the total amount of pixels of the image. 
In all cases, the difference between the results obtained using the ABC-ED algorithm and the Canny algorithm is zero. Please note that both algorithms consider the same threshold values.

Table 3 shows the results (best outputs) when the criteria is to minimise the number of pixels to analyse $(P A)$. The difference between the ABC-ED algorithm and Canny is zero (both cases consider the same threshold). In Table 3 , the first row (less percentage of pixels to analyse) shows that the ABC-ED algorithm requires $10.24 \%$ of pixels analysed, and 57 execution cycles. The difference of the output image with respect to the ground truth image $\Delta \mathrm{GT}$ is $0.78 \%$.

Table 3. The Best Results - Pixel Analysis

\begin{tabular}{|l|c|c|c|}
\hline Image & Number of cycles & PA (\%) & $\Delta$ GT (\%) \\
\hline 1 & 57 & 10.24 & 0.78 \\
\hline 2 & 52 & 10.29 & 0.5 \\
\hline 3 & 55 & 10.36 & 0.79 \\
\hline 4 & 57 & 10.48 & 0.58 \\
\hline 5 & 58 & 10.55 & 0.79 \\
\hline 6 & 70 & 10.59 & 0.6 \\
\hline 7 & 74 & 10.63 & 0.91 \\
\hline 8 & 54 & 10.67 & 0.58 \\
\hline 9 & 58 & 10.68 & 0.59 \\
\hline 10 & 51 & 10.72 & 0.56 \\
\hline
\end{tabular}

Figure 1 shows an example of two original images taken from the dataset used for testing.

\section{Figure 1. Example of Two Original Images}
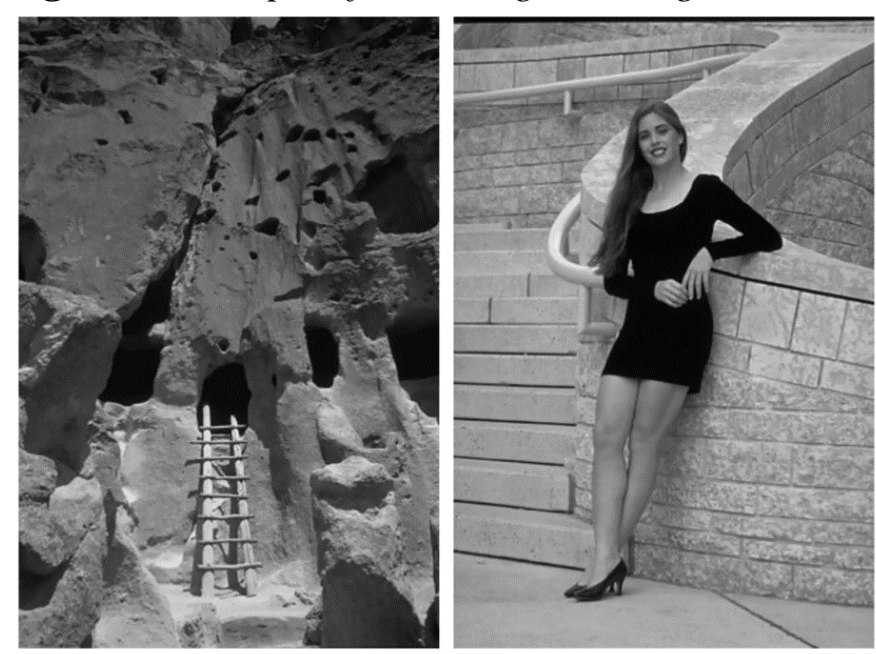

Figure 2 shows one of the possible segmentations of the images shown in Figure 1. 
Figure 2. Example of Two Segmented Images

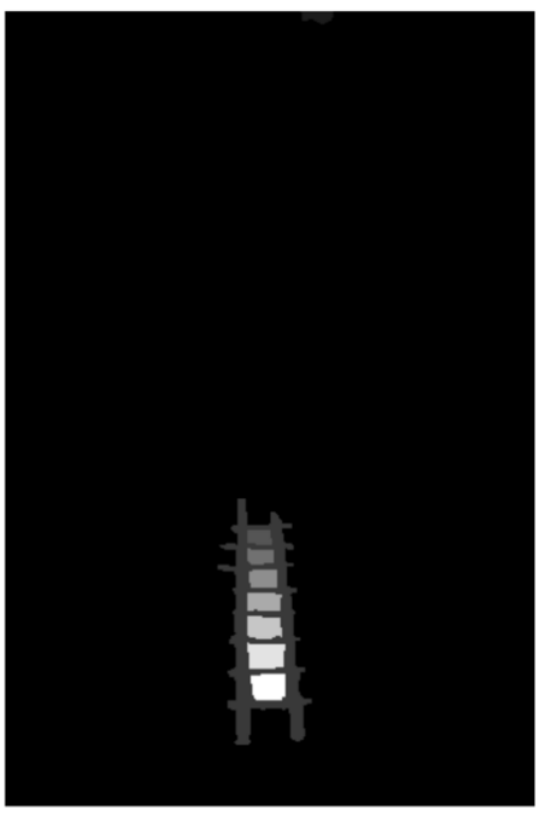

(a)

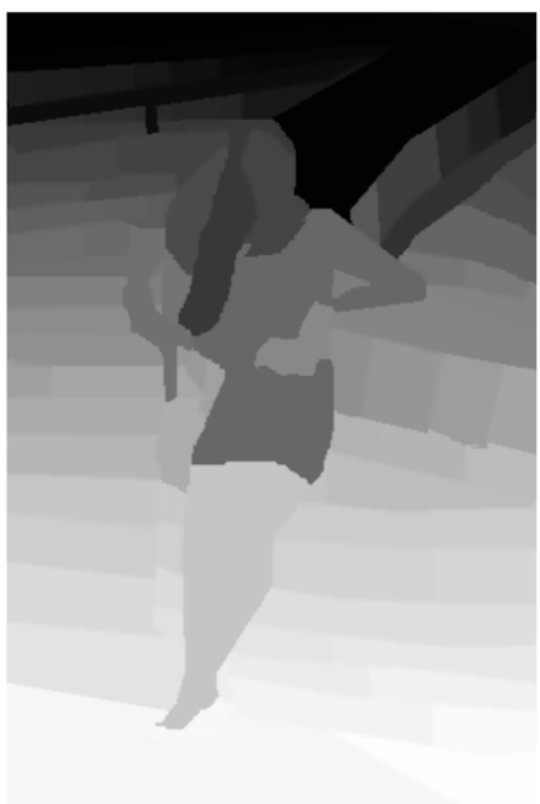

(b)

Figure 3 shows the corresponding ground truth of the segmented images in Figure 2.

Figure 3. Example of two Ground Truth Images
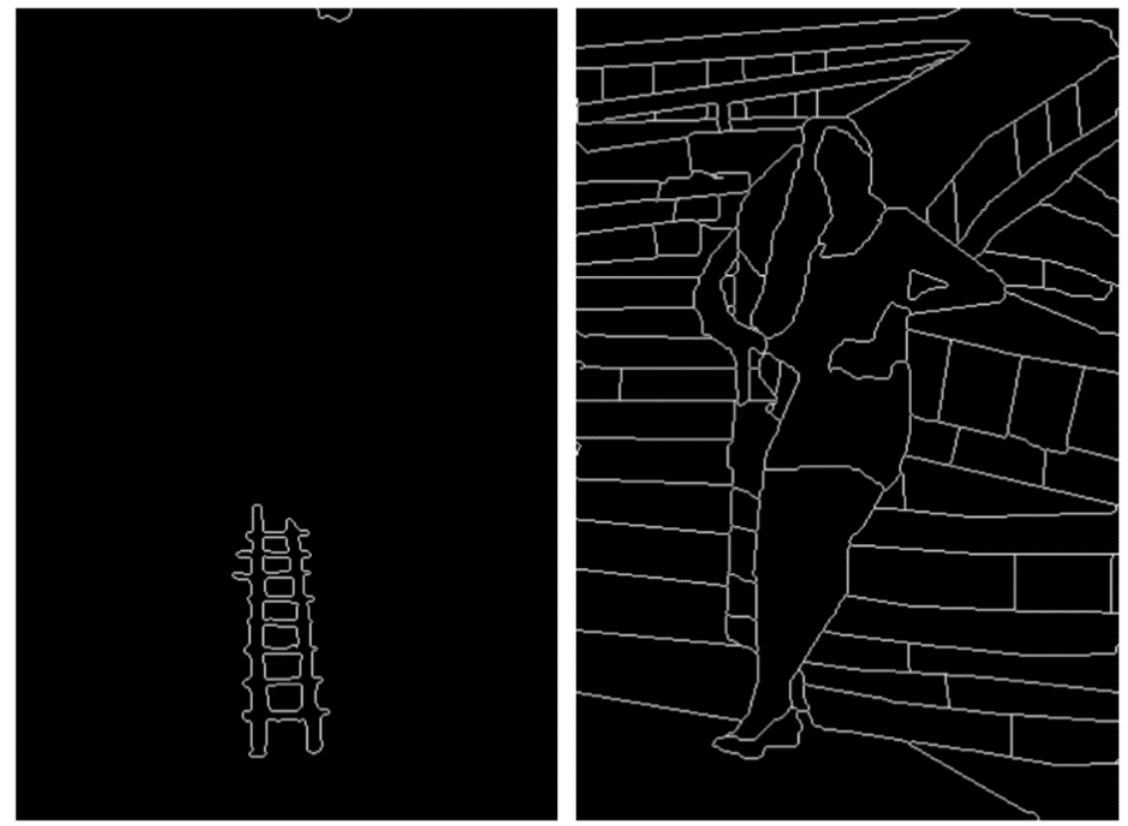

Figure 4 shows the output images obtained by applying the ABC-ED algorithm to the images of Figure 2. Please note there are small differences between Figure 3 and Figure 4. 
Figure 4. Example of Two Output Images

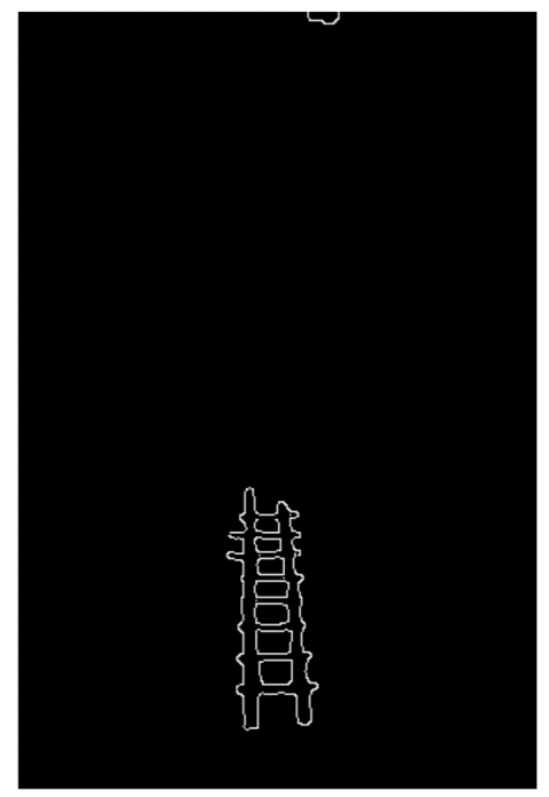

(a)

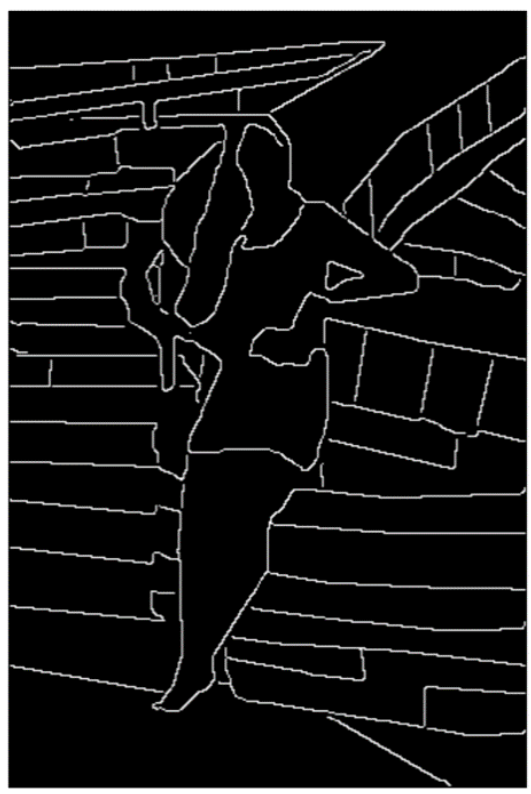

(b)

It is important to note that the input to the ABC-ED algorithm is a segmented image, (see Figure 2). This is because in the chosen database, the available ground truth images refer to segmented images (not to the original images).

Different images require different number of pixels to analyse. If edges are homogeneously distributed on the whole image, as in Figure $2 b$, the behaviour of the algorithm is similar to Canny performance. This is because the algorithm needs to analyse over $95 \%$ of pixels. The difference of the output image with respect to the ground truth image is close to $2 \%$. Figure $2 \mathrm{~b}$ belongs to the second group of images in Table 1 . In the case of Figure $2 b$, the result is the output image using our approach, is shown in Figure $4 b$. If there are regions in an image with no edges, then the performance of the ABC-ED algorithm is improved.

Figure $2 \mathrm{a}$ belongs to the first group of images in Table 1. For this image, $10.29 \%$ of the pixels of the image are analysed. In the case of Figure 2a the result is the output image shown in Figure 4a. The difference with respect to the ground truth image is $0.5 \%$. 
Figure 5. Original Image and Corresponding Output
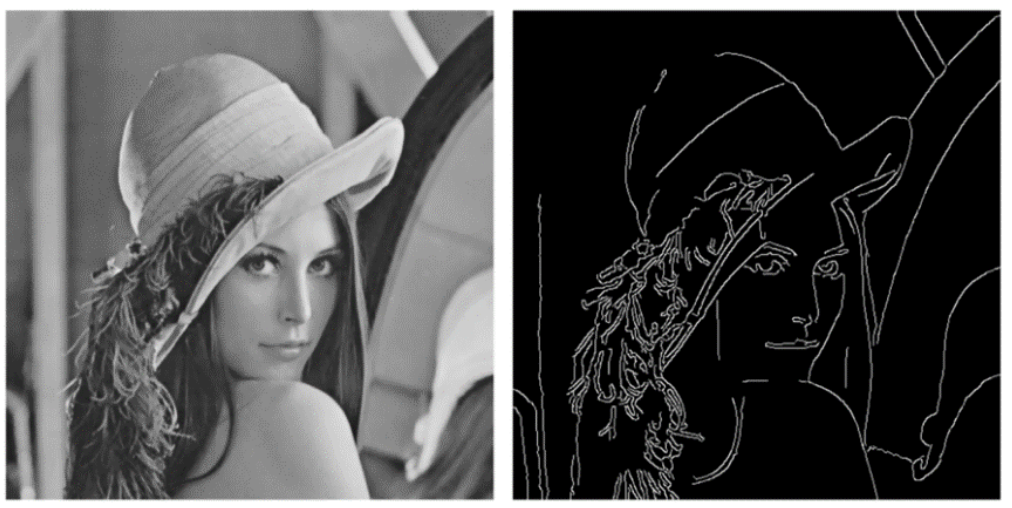

Figure 5 shows an example for a typical image (Lenna) and the resulting image using the ABC-DE algorithm. More specifically, the left side of shows the original image and right side, shows the result using the ABC-ED algorithm.

Similarly, Figure 6 shows another typical image example (Cameraman). On the left the original image (Cameraman) and on the right the result using the ABC-ED algorithm.

Figure 6. Original Image and Corresponding Output
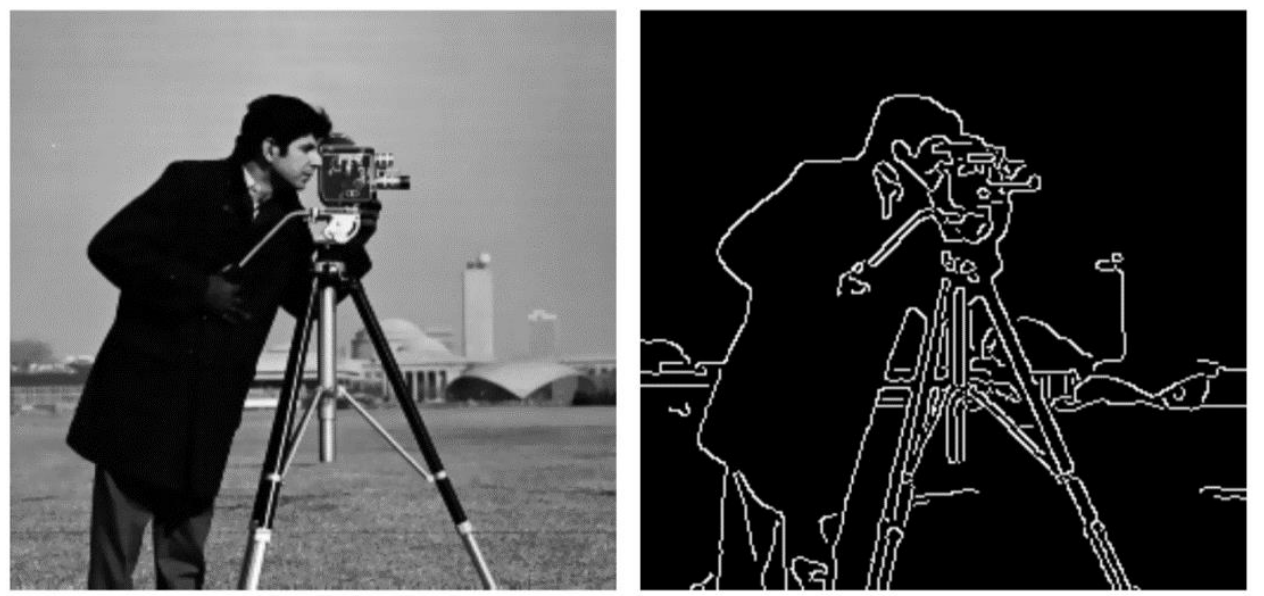

For the above images, the results are the same as the results obtained using the Canny algorithm, Otsu method (Otsu 1979). In the case of Figure 5 , it is necessary to analyse $33.62 \%$ of the pixels. In the case of Figure 6 , it is necessary to analyse $34.96 \%$ of the pixels.

\section{Conclusions}

This work integrates the $\mathrm{ABC}$ algorithm and the Canny algorithm. The result, the $\mathrm{ABC}-\mathrm{ED}$ algorithm, reduces the number of pixels to analyse in an image, to detect its edges. 
Results show that changes introduced to the $\mathrm{ABC}$ algorithm, and its combination with Canny algorithm, achieve the objectives proposed for this new algorithm: to detect every edge that Canny detects, avoiding analysing all pixels in the original image. Due to the fact that the algorithm integrates the Canny edge identification, it detects every edge that Canny algorithm detects.

The BSDS500 dataset was used in order to analyse the algorithm performance. The algorithm performs better when analysing images bounded to specific regions. Performance decreases for images with homogeneously distributed edges. The average value for PA is less than $60 \%$, when all the images in the dataset are taken into account.

Additionally, experiments show that the number of pixels to analyse depends more on the image than the mechanism used to detect edges. The proposed algorithm obtains results that (in average) differ from ground truth values in no more than $2.01 \%$.

\section{References}

Adelson-Velskii GM, Landis EM (1962) An Algorithm for the Organization of Information. Dokladi Akademia Nauk SSSR 146(2): 1259-1262.

Ahmad R, Akhtar, N, Choubey N (2017) Applications of Artificial Bee Colony Algorithms and its Variants in Health Care. Bio-Chemistry: An Indian Journal 11(1): 110.

Baeck T, Fogel DB, Michalewicz Z (2000) Evolutionary Computation 1: Basic Algorithms and Operators. Bristol: Institute of Physics Publishing and New York: Oxford University Press.

Basturk B, Karaboga D (2006) An Artificial Bee Colony (abc) Algorithm for Numeric Function Optimization. In IEEE Swarm Intelligence Symposium 8: 687-697.

Benala TR, Jampala SD, Villa SH, Konathala B (2009) A Novel Approach to Image Edge Enhancement using Artificial Bee Colony Optimization Algorithm for Hybridized Smoothening Filters. In Nature \& Biologically Inspired Computing. NaBIC 2009: 1071-1076.

Canny J (1986) A Computational Approach to Edge Detection. In Transactions on Pattern Analysis and Machine Intelligence 6: 679-698.

Cuevas E, Osuna V, Oliva D (2017) Evolutionary Computation Techniques: A Comparative Perspective. New York, NY: Springer.

Deng Y, Duan H (2014) Biological Edge Detection for UCAV via Improved Artificial Bee Colony and Visual Attention. In Aircraft Engineering and Aerospace Technology: An International Journal 86(2): 138-146.

Glasbey CA (1993) An Analysis of Histogram-based Thresholding Algorithms. CVGIP: Graphical Models and Image Processing 55(6): 532-537.

Hamming RW (1950) Error detecting and error correcting codes. Bell System technical journal 29(2): 147-160.

Karaboga D (2005) An Idea Based on Honey Bee Swarm for Numerical Optimization. Retrieved from https://bit.ly/2Zh2SPS.

Karaboga D, Akay B (2009) A Comparative Study of Artificial Bee Colony Algorithm. Applied Mathematics and Computation 214(1): 108-132.

Martin D, Fowlkes C, Tal D, Malik J (2001) A Database of Human Segmented Natural Images and its Application to Evaluating Segmentation Algorithms and 
Measuring Ecological Statistics. Proceedings Eighth IEEE International Conference on Computer Vision 2: 416-423.

Moore GA (1969) Automatic Scanning and Computer Processes for the Quantitative Analysis of Micrographs and Equivalent Subjects. Pattern Recognition: Pictorial Pattern Recognition 1: 275-326.

Otsu N (1979) A Threshold Selection Method from Grey-level Histograms. IEEE Transactions on Systems, Man and Cybernetics 9(1): 62-66.

Parmaksizoglu S, Alci M (2011) A Novel Cloning Template Designing Method by using an Artificial Bee Colony for Edge Detection of cnn Based Imaging Sensors. Sensors 11: 5337-5359.

Prewitt J (1970) Object Enhancement and Extraction. Picture Processing and Psychopictorics 10(1): 15-19.

Roberts LG (1965) Machine Perception of Three Dimensional Solids. Cambridge: M.I.T. Lincoln Laboratory.

Sobel I, Feldman G (1968) A 3x3 Isotropic Gradient Operator for Image Processing. Pattern Classification and Scene Analysis 271-272.

Thereshko V (2000) Reaction-Diffusion Model of a Honeybee Colony's Foraging Behaviour. Parallel Problem Solving from Nature, PPSN VI 1917: 807-816.

Torre V, Poggio TA (1986) On Edge Detection. IEEE Transactions on Pattern Analysis and Machine Intelligence 8(2): 147-163.

Yigitbasi E, Baykan N (2013) Edge Detection Using Artificial Bee Colony Algorithm (ABC). International Journal of Information and Electronics Engineering 3(6): 634-638.

Ziou D, Tabbone S (1998) Edge Detection Techniques - An Overview. Pattern Recognition \& Image Analysis 8(4): 537-559. 